\title{
Kernos
}

Revue internationale et pluridisciplinaire de religion grecque antique

12 | 1999

Varia

\section{R. HÄGG (éd.), The Role of Religion in the Early Greek Polis}

\section{Madeleine Jost}

ERL : http://journals.openedition.org/kernos/732

DOI : $10.4000 /$ kernos.732

ISSN : 2034-787

\section{Éditeur}

Centre international d'étude de la religion grecque antique

\section{Édition imprimée}

Date de publication : 1 janvier 1999

Pagination : 305-307

ISSN : 0776-3824

Référence électronique

Madeleine Jost, «R. HÄGG (éd.), The Role of Religion in the Early Greek Polis », Kernos [En ligne], 12 |

1999, mis en ligne le 13 avril 2011, consulté le 11 mars 2021. URL : http://journals.openedition.org/ kernos/732 ; DOI : https://doi.org/10.4000/kernos.732 
le soldat mort à Eleutherna serait un défunt de haut rang, et aurait bénéficié d'un rituel organisé. Il aurait probablement été tué par l'homme décapité, un personnage de rang inférieur. Le dernier aurait été condamné à être décapité sur la tombe de sa victime et mutilé en ses extrémités [p. 185-189]. Le dernier chapitre de l'exposé reprend la bibliographie sur les croyances grecques relatives à l'âme.

En conclusion, l'A. propose une hypothèse qui semble concilier les données archéologiques. Il s'agit de faire de la tombe d'une personne de haut rang le lieu d'exécution d'une autre personne [p. 201]. Cette tentative d'interprétation paraît plausible, mais on pourrait émettre quelques réserves sur les détails : en effet, l'ensemble des indications de la fouille offre une image plutôt floue de la fonction du site comme lieu de représailles. Dans l'état actuel de nos connaissances, il est difficile de voir dans le squelette sans tête d'Eleutherna le résultat d'une structure sociale particulière ou d'envisager l'existence généralisée d'un traitement des prisonniers de guerre. En l'absence de solution immédiate et d'éléments de comparaison archéologiques, il faudrait sans doute se limiter à des hypothèses plus prudentes.

La variêté et la qualité des illustrations du livre sont remarquables. La consultation des illustrations est facilitée par une liste des figures. L'ouvrage est complété par la bibliographie et un index.

Eleni Georgoulaki (Athènes)

Robin HägG (éd.), The Role of Religion in the Early Greek Polis. Proceedings of the Third International Seminar of Ancient Greek Cult, organized by the Swedish Institute at Athens, 16-18 October 1992, Stockholm, Åström, 1996. 1 vol. $16,5 \times 24,2 \mathrm{~cm}, 176$ p. , ill. (Acta Instituti Atbeniensis Regni Sueciae, Series in-8 $\left.{ }^{\circ}, 14\right)$. ISBN : 91-7916-033-6.

Contrairement aux deux volumes précédents, qui étaient axés, l'un sur l'iconographie ${ }^{1}$, l'autre sur l'épigraphie ${ }^{2}$, cette publication du troisième séminaire international sur les cultes anciens de la Grèce est pluridisciplinaire, faisant intervenir l'archéologie, l'iconographie, la littérature et l'épigraphie. L'objet est d'étudier le rôle de la religion lors de la formation et des débuts de l'histoire de la polis. Les contributions abordent des sujets très variés, dont on aura ici un aperçu. - Irad Malkin ( "The polis between myths of land and territory », p. 9-19) s'intéresse à l'interférence du mythe et de la politique dans la polis primitive. L'essentiel de l'article porte sur la légende libyenne de la victoire d'Héraklès sur Antée : elle reflète l'ouverture du pays à la civilisation et ne cesse, pendant deux siècles, de s'étendre vers l'Est, selon les acquisitions territoriales et les fondations coloniales, au fur et à mesure de l'extension du territoire de Cyrène. - Walter Burkert ("Greek temples-builders: who, where and why? ", p. 21-29) souligne le fait que l'édification d'un temple est d'abord une démonstration de prestige, en forme de remerciement aux dieux (le butin de guerre doit avoir joué dès les premiers temps un rôle important dans le financement des monuments); il insiste ensuite sur deux motifs religieux: la compensation d'un acte impie et la réponse à un oracle ou à des signes divins. Au total, le hasard, prérogative des dieux, et le choix des hommes se conjuguent pour créer le paysage religieux d'une cité. -

1 R. HägG (éd.), The iconograpby of Greek cult in the Archaic and Classical periods, Liège-Athènes, 1992 (Kemos, suppl. 1).

2 R. Ḧ̈GG (éd.), Ancient Greek cult practice from the epigrapbical evidence, Stockholm, 1994 (ActaAtb-8, 13 ). 
François de Polignac ( Entre les dieux et les morts. Statut individuel et rites collectifs dans la cité archaïque », p. 31-40) revient sur l'idée exprimée par divers auteurs, selon laquelle le début de l'archaïsme serait marqué par un transfert d'offrandes, des sépultures aux sanctuaires. Il convient, dit-il, de nuancer cette opinion, au vu des données de l'archéologie en Argolide et en Attique qui suggèrent l'élaboration d'une "nouvelle articulation entre les deux domaines »: il n'y a pas d'évolution séparée du privé et du public. - Sanne Houby-Nielsen ("The archaeology of ideology in the Kerameikos: new interpretation of the "Opferrinnen" », p. 41-54) étudie les tranchées à offrandes des tombes du viI ${ }^{\mathrm{e}}$ siècle. La céramique retrouvée ne provient pas du banquet funéraire, mais elle résulte d'offrandes faites pour exprimer la qualité du mort, qui préfigurent la célébration des valeurs aristocratiques sur les reliefs funéraires (noter des schémas très parlants p. 45 et 53). - Fritz Graf ( Pompai in Greece. Some considerations about space and ritual in the Greek polis», p. 55-65) étudie les différentes formes de processions, centripètes et centrifuges, ordonnées (Grandes $\mathrm{Pa}$ nathénées) ou désordonnées (Grandes Dionysies). Ces manifestations permettent de définir les relations entre un sanctuaire donné, ses cultes et ses divinités, et la polis. L'A. termine par le cas plus complexe d'Éleusis. - Allaire Brumfield, "Aporreta: verbal and ritual obscenity in the cults of ancient women », p. 67-74) aborde la question de ces mots qui ne «doivent pas être dits ", à la fois parce qu'ils sont secrets et parce qu'ils sont réputés indécents, et que prononçaient les femmes pendant les mystères de Déméter. Ils expriment, pour l'auteur, un «discours féminin » qui témoigne du pouvoir des femmes dans le rituel. - Patricia A. Butz, ("Prohibitionnary inscriptions, $\xi \in \epsilon \nu O L$, and the influence of the early Greek polis», p. 75-95) étudie quatre inscriptions de Délos, Paros, Amorgos et Mykonos qui interdisent l'accès de lieux sacrés aux étrangers. Elle insiste sur le rôle de l'exclusion pour définir un groupe social. La date des textes cités (le plus ancien est du milieu du $v^{e}$ siècle) empêche néanmoins toute certitude quant aux origines de la cité. - Jan Bremmer ( "The status and symbolic capital of the seer», p. 97-109) s'intéresse au statut du devin dans la cité et à son évolution au cours de l'histoire; il note comment l'expédition de Sicile en 413 av. J.-C. affecta durablement la réputation des devins. - Kevin Clinton ( « The Thesmophorion in central Athens and the celebration of the Thesmophoria in Attica », p. 111-125) démontre que la célébration des Thesmophories n'était pas organisée par Athènes, mais par les dèmes où la fête se déroulait. La cérémonie décrite par Aristophane peut avoir été organisée par le dème de Mélité. Il s'agirait, dans les sources qui ne sont pas antérieures au IV siècle, d'une survivance du rôle des dèmes avant le synoecisme d'Athènes. - H.A. Shapiro ("Cults of Solonian Athens", p. 127-133) compare, grâce à Plutarque surtout, les traditions relatives à Solon et à Thésée. Le parallélisme serait dû à ce que les deux héros ont eu très tôt un statut comparable de " héros de la culture ». - Nanno Marinatos ( Cult by the seashore: what happened at Amnisos? », p. 135-139) revient sur la grotte dite d'Eileithyia à Amnisos. Des comparaisons entre ce sanctuaire maritime et la grotte d'Inatos en Crète ou l'Héraion à Samos suggèrent que ces sanctuaires de bord de mer étaient multifonctionnels, entre des cultes locaux et des cultes cosmopolites. - Marcel Piérart ( « La mort de Dionysos à Argos », p. 141-151) démontre que Déméter et Dionysos ne forment pas un couple à Lerne, comme le voulait Nilsson. Les Lernaia étaient des mystères de Déméter. Le mythe de l'arrivée de Déméter, tel qu'on le racontait à Argos, était lié à Pélasgos et au cycle d'Inachos. Au contraire, Dionysos était lié au cycle de Persée, qui l'aurait mis à mort; son culte à Lerne comportait des rites particulièrement anciens. À Argos, on avait conservé dans les monuments le souvenir de la lutte entre Persée et Dionysos, ensuite réconciliés. Déméter avait des monuments dans le secteur Nord-Est de la ville. - Au total, ce livre est dans sa 
diversité même d'une grande richesse; il montre aux historiens des religions ce que peuvent apporter les sources les plus diverses pour l'étude d'une période qui est largement pré-littéraire.

Madeleine Jost

(Paris X-Nanterre)

Fritz GraF (éd.), Ansichten griechischer Rituale. Geburtstags-Symposium für Walter Burkert. Castelen bei Basel 15. bis 18. März 1996, Leipzig, Teubner, 1998.1 vol. $16 \times 24 \mathrm{~cm}$, vi+467 p., 39 fig. ISBN : 3-519-07433-8.

Célébrer Walter Burkert, c'est fêter un savant qui a profondément marqué les études sur la religion grecque antique ces trente dernières années, avec des apports fondamentaux sur le rituel, et le sacrifice en particulier. C'est donc autour de ses thèmes de prédilection que se noue ce recueil articulé en quatre grandes parties.

La première (Grundlagen und Reflexionen) aborde les notions de "religion », de «rituel», de «sacré » et de «profane» dans une remarquable perspective historiographique (J.N. Bremmer), et les problèmes touchant à la compréhension difficile du rituel grec au sens large, avec l'accent porté in fine sur l'Homo Necans de Burkert (A. Henrichs). Une étude sur la figuration de scènes violentes dans la céramique attique entre 550 et 450 av. J.-C., intéressante du point de vue de la sacralisation et de la ritualisation de la terreur, termine cette partie ( $\mathrm{P}$. Blome).

La deuxième partie (Riten in der Geschichte). propose huit contributions variées, mêlant (1) un panorama du rituel dans la Grèce mycénienne (R. Hägg), (2) une (trop) brève étude sur l'iconographie et la personnalité d'Artémis (N. Marinatos), (3) une approche archéologique de la libation et de la prière gréco-romaines (E. Simon), - où l'on aurait aimé voir discutés l'article de P. Veyne (Mètis, 5 [1990], p. 17-30) et le livre de D. Aubriot (cf. Kernos, 6 [1993], p. 379-381), - (4) une approche résolument «agraire» du rituel initiatique patréen pour Artémis et Dionysos rapporté par Pausanias (G. Baudy), - qui tranche avec les interprétations anthropologiques récentes de ce type d'initiation, - (5) une excellente réévaluation du rapport entre ritus romanus et ritus graecus dans la pratique romaine (J. Scheid), (6) et une autre de la définition du taurobolion, dont la fossa sanguinis est une invention d'auteurs chrétiens (Ph. Borgeaud), (7) une analyse critique des calendes de janvier comme "fête de dissolution et de renouveau » dans l'empire romain (Fr. Graf), et enfin (8) une étude fouillée des defixiones anatomiques (H.S. Versnel).

La troisième partie rassemble trois contributions autour du thème Ritual und Tragödie, en écho au célèbre article de Burkert Greek Tragedy and Sacrificial Ritual. H. Lloyd-Jones, après une introduction personnalisée, conteste l'omniprésence, dans la tragédie, des rituels, sacrificiels ou autres, et donc la permanence des liens du genre avec ses origines anciennes. E. Krummen étudie davantage l'utilisation métaphorique du rituel par la tragédie, mais rejoint ses conclusions sur l'origine du genre. L'analyse de l'Edipe à Colone par Cl. Calame montre Sophocle réinterprétant le processus symbolique, entre «mythe » et « rite ».

La dernière partie (Orpbica et Philosophica) est centrée sur un autre domaine de prédilection de W. Burkert et aborde les questions de l'énonciation dans lamelles orphiques (Chr. Riedweg) et de l'anthropologie qui s'en dégage (H.D. Betz). Elle se referme sur une analyse de l'évolution des conceptions de la $\tau$ tún due aux dieux depuis Hésiode et les Présocratiques jusqu'à Platon et Aristote.

Vinciane Pirenne-Delforge (FNRS - Université de Liège) 